\title{
Desajuste educacional y de competencias: efectos diferenciales sobre la satisfacción laboral. Un estudio aplicado al mercado de trabajo español*
}

Educational and skill mismatches: differential effects on job satisfaction. A study applied to the Spanish job market

\author{
NuRIa SÁNCHEZ-SÁNCHEZ** \\ Adolfo C. Fernández Puente****
}

\begin{abstract}
Resumen
El objetivo de este trabajo es estudiar el efecto de los desajustes educacionales y de habilidades en la satisfacción de los trabajadores en su puesto de trabajo, globalmente y respecto del salario, el número de horas, las posibilidades de promoción y el tipo de actividad desempeñada. Para ello se estima un modelo probit, utilizando datos procedentes de la Encuesta de Calidad de Vida en el Trabajo (ECVT). Los resultados muestran que los individuos con un nivel educacional superior al exigido por el puesto de trabajo se encuentran más insatisfechos que aquellos en los que el ajuste es adecuado. Sin embargo, en el caso de los trabajadores con un nivel educacional inferior al que requiere su trabajo, la pérdida de satisfacción no resulta significativa. No ocurre lo mismo con los desajustes por habilidades en los que se evidencia que aquellos individuos con habilidades inferiores a las exigidas están más insatisfechos que los que tienen una correcta adecuación. Esta disparidad en los resultados evidencia la necesidad de estudiar ambos desajustes, educacional y de competencias, por separado.
\end{abstract}

Palabras clave: Satisfacción laboral, ajuste educacional, ajuste de habilidades.

Clasificación JEL: J24, J31.

\begin{abstract}
This paper focuses on the effect of educational and skill mismatches of workers on overall job satisfaction, and on certain job domains such as salary, number of working hours, promotion opportunities and type of activity performed. An

\footnotetext{
Los autores agradecen los valiosos comentarios realizados por dos árbitros anónimos de la revista Estudios de Economía, los que contribuyeron sustancialmente a la mejora de versiones preliminares del presente trabajo.

**Universidad de Cantabria. Email: sanchezn@unican.es

***Universidad de Cantabria. Email: adolfo.cosme@unican.es
} 
ordered probit model is estimated by using a data set from the Survey of Quality of Working Life (ECVT). In addition to the variables related to these mismatches, other variables related to the characteristics of both the individual and the type of work are included. The results show that overeducated individuals show higher levels of dissatisfaction than those in which the fit is adequate. However, it also shows how individuals with a lower level of education than what would be required for the post do not reflect significant dissatisfaction levels. It is also evidence observed that individuals with less than the required skills are more dissatisfied than those with a proper matching. The results support the need to study both educational and skill mismatches separately.

Key words: Satisfaction, educational match, skill match.

JEL Codes: J24, J31.

\section{INTRODUCCIón}

El nivel educacional de los trabajadores españoles se ha incrementado de forma sustantiva en los últimos 50 años. En 1964 el $93 \%$ de la población en edad de trabajar carecía de estudios, o solo había completado los estudios primarios, y $11,9 \%$ era analfabeta (Pérez et al., 1998), mientras que en 2012 estos porcentajes eran del $28,4 \%$ y $2,1 \%$, respectivamente ${ }^{1}$. La alta inversión en capital humano ha permitido, de hecho, incluir a España dentro del grupo de países de la OCDE con mayores tasas de licenciados universitarios entre la población más joven. La demanda de estudios universitarios ha crecido a una mayor tasa que la oferta de puestos de trabajo, de forma que en la actualidad se observan considerables desajustes educacionales entre los trabajadores y los puestos de trabajo que ocupan. Este hecho es compatible con desajustes en sentido inverso, es decir, el grupo de trabajadores que poseen un nivel de estudios por debajo del requerido por su puesto. Si bien, la cuantía relativa de este último grupo, tal y como se desprende de los datos, es más reducida.

Gran parte del desajuste educacional obedece al desfase temporal entre las decisiones educativas y la incorporación de los jóvenes al mercado laboral propias de una economía que está experimentando cambios estructurales sustanciales, como la española (Albert y Mallorquí, 2011). La elevada demanda de estudios de nivel superior por parte de las mujeres, unida a los altos niveles de desempleo padecidos por la economía española en diversos períodos de las dos últimas décadas intensifica este desajuste. Como muchos jóvenes no encuentran trabajo dentro del nivel educacional alcanzado, tienen que aceptar trabajos de uno inferior. Si la hipótesis de Albert y Mallorquí (2011) fuese cierta, a medida que la economía alcanzase un nivel de renta equiparable a la media europea este problema disminuiría. Sin embargo, existen indicios para pensar de que no se trata de un problema transitorio exclusivamente, sino permanente (García Serrano y Malo, 1996). En España, el porcentaje de trabajadores sobreeducados

1 Encuesta de Población Activa del Instituto Nacional de Estadística (INE). 
se sitúa en torno al 25\%, mientras que la media europea lo hace en torno al $22 \%{ }^{2}$ (Budria y Moro-Egido, 2009).

Además del desajuste educacional, la literatura económica centra sus esfuerzos en otro tipo de desajuste laboral, el de habilidades, capacidades o competencias $^{3}$. Este último se asocia a las diferencias que pueden producirse entre las habilidades de un trabajador y las requeridas para el correcto desempeño de su puesto de trabajo. Dichas capacidades en algunos casos pueden ser innatas y en otros pueden ser adquiridas por la experiencia y la formación en el puesto de trabajo. Nuevamente, al igual que en el caso de la educación, se pueden encontrar trabajadores con capacidades superiores, iguales o inferiores a las del puesto de trabajo que ocupan. La evidencia empírica reciente pone de manifiesto la importancia de tener en cuenta ambos tipos de desajustes a la hora de hacer una valoración idónea de sus consecuencias económicas, debido a que el capital humano de un trabajador no está constituido exclusivamente por su educación, sino también por sus habilidades.

La hipótesis que contrastamos es que aquellos trabajadores que han invertido recursos (dinero y tiempo) en recibir una educación se encontrarían menos satisfechos si no pudieran alcanzar un puesto acorde con ese nivel educacional. Por otra parte, cabría esperar un grado de satisfacción superior en el caso de aquellos trabajadores que no hayan estudiado pero alcancen un puesto donde normalmente se sitúan trabajadores más formados. En lo que respecta al ajuste de habilidades, el grado de satisfacción debiera ser menor para aquellos trabajadores con habilidades inferiores a las exigidas en el puesto, ya que encuentran más dificultades para realizar un correcto desempeño de su trabajo.

La novedad del enfoque propuesto en el artículo radica en tres aspectos. En primer lugar, el número de estudios que abordan el efecto del desajuste educacional y de competencias sobre la satisfacción, de forma conjunta, es muy reducido en España ${ }^{4}$. En segundo lugar, la ECVT no solo permite identificar la influencia del desajuste educacional sobre la satisfacción laboral global, sino también sobre otros aspectos del trabajo como el salario, la jornada de trabajo, la promoción, y el tipo de trabajo. En tercer lugar, la riqueza de la encuesta en lo referente a variables subjetivas, como el estrés, el ambiente laboral o la independencia del trabajo, hace posible evitar sesgos que puedan sobrevalorar el impacto de los desajustes laborales.

El trabajo se estructura de la siguiente forma. En el segundo apartado, y como marco de análisis, se hace una breve descripción de la literatura que relaciona los desajustes educacionales y de habilidades sobre la satisfacción. En el tercero, se describen los datos utilizados y la interpretación de las respuestas de la ECVT. En el cuarto y quinto apartado se define el modelo econométrico y se realizan las estimaciones. Finalmente, se concluye.

2 Estas cifras no coinciden exactamente con las aportadas por la ECVT utilizadas en este artículo, lo que se debe a la disparidad de metodologías utilizadas. La ECVT no aporta los datos a nivel europeo que permita hacer comparaciones.

3 De aquí en adelante se utilizarán indistintamente los tres términos.

4 Véase la sección 2 referente a la literatura que liga los desajustes educacionales y de competencias con la satisfacción. 


\section{Literatura}

Los desajustes educacionales suponen una ineficiencia en la asignación de los recursos y pueden dar lugar a una merma de la productividad del trabajador y, por ende, de los resultados de la empresa (Tsand y Levin, 1985). La influencia de la sobreeducación en la productividad se canalizaría mediante distintos mecanismos: el ausentismo, las bajas voluntarias, el grado de rotación del empleo y la satisfacción laboral (Peiró et al., 2010; Battu et al., 1999; Fleming and Kler, 2008; Hersch, 1991; Tsang, 1987, Tsang and Levin, 1985; Verhaest and Omey, 2006). Por otra parte, la literatura existente señala que los trabajadores sobreeducados tienen un salario relativo inferior al que les correspondería en caso de desempeñar un puesto acorde con su nivel educacional, esto es un motivo adicional de insatisfacción ${ }^{5}$. McGuinness y Sloane (2011) señalan que no solo los sobreeducados, sino también los infraeducados exhiben una menor satisfacción laboral.

En principio, si se produjese un solapamiento entre los individuos que presentan desajustes educacionales y de competencias, los resultados sobre la satisfacción debieran ser similares, aunque la evidencia empírica parece no confirmar esta hipótesis (Green y McIntosh, 2007). Un individuo infracapacitado tendrá una productividad inferior a la de otros trabajadores con capacitación adecuada al puesto y, por tanto, es factible que tenga un nivel salarial inferior. Por lo que respecta a la satisfacción, esta podría ser inferior, no solo por los niveles salariales, sino porque es factible que necesite más tiempo para realizar las mismas tareas que otros trabajadores y su nivel de estrés será superior. No obstante, los estudios empíricos no son concluyentes. Por ejemplo, Allen y Van der Velden (2001) y Cabral (2005) muestran que el desajuste en capacidades tiene un efecto negativo en la satisfacción laboral. McGuinness y Sloane (2011) señalan que aquellos individuos que están ocupando puestos que exigen un nivel de competencias inferior al que poseen están menos satisfechos que aquellos en los que se produce un ajuste adecuado ${ }^{6}$. Considerando ambas variables de forma conjunta, Green y Zhu (2010) concluyen que la sobreeducación no es un problema para la satisfacción laboral si no va acompañada con desajuste en habilidades.

Los trabajos realizados para España son escasos y sus conclusiones dispares. Peiró et al. (2010), y Fabra y Camisón (2008) señalan que existe una relación negativa entre sobreeducación y satisfacción. Sin embargo, Badillo-Amador et al. (2012) muestran lo contrario, presentando evidencia de que el desajuste educacional tiene un efecto neutral e incluso positivo en la satisfacción laboral (particularmente en trabajadores infraeducados), y que es únicamente el desajuste en habilidades, tanto por exceso como por defecto, el que tiene un impacto negativo. Por su parte, McGuiness y Sloane (2011) encuentran que únicamente los trabajadores con habilidades inferiores a las exigidas en el puesto de trabajo están menos satisfechos en su trabajo. Las discrepancias obtenidas en los

5 Freeman (1976) fue uno de los primeros en estudiar las consecuencias económicas de la sobreeducación en los salarios relativos. Para una revisión más actual de literatura que relaciona sobreeducación con el resto de las variables véase McGuiness (2006).

6 Viceversa en el caso de ocupar un puesto que exige un nivel de habilidades superior. 
estudios realizados sobre la relación entre los desajustes educacionales y de habilidades y la satisfacción laboral permiten entrever la necesidad de abordar nuevos estudios que ayuden a clarificar esta relación.

\section{Datos}

Los datos utilizados en este estudio provienen de la ECVT. Esta encuesta toma como muestra a más de 7.000 trabajadores y recoge información sobre empleo, movilidad, condiciones de trabajo y opinión del trabajador, entre otros aspectos ${ }^{7}$. El marco temporal del estudio abarca el período comprendido entre 2006 y 2008, si bien la encuesta no es un panel, ya que no se recogen los mismos individuos cada año. La ECVT proporciona varias ventajas añadidas respecto de otras encuestas utilizadas en trabajos anteriores. En primer lugar, no contempla exclusivamente la satisfacción laboral global, sino la correspondiente al salario, al tipo de trabajo, al número de horas, a la flexibilidad laboral, y a la promoción, entre otros aspectos relacionados con el trabajo. En segundo lugar, la calidad de los datos permite tener en cuenta, no solo las características del trabajador (edad y sexo) y las características objetivas del puesto de trabajo (salario y tipo de puesto), sino otras de carácter más subjetivo relacionadas con el entorno laboral, el desarrollo de tareas creativas, la autonomía, las responsabilidades asumidas, el estrés y la relación con los superiores e inferiores. Este tipo de variables reflejan beneficios no salariales para el trabajador que pueden estar influyendo significativamente en la satisfacción laboral.

La medición de la variable relacionada con el desajuste educacional se puede realizar por medio de tres procedimientos:

i) La medida objetiva: supone una evaluación sistemática para determinar el nivel educacional requerido para un determinado trabajo, el que se compara con el del trabajador. El trabajador se define como sobreeducado si ha realizado más años de estudio de lo que requiere su puesto de trabajo (viceversa en el caso de infraeducado).

ii) La medida subjetiva: se basa en la respuesta que da el trabajador a la pregunta de cuántos años de estudios se requieren para conseguir un trabajo como el suyo. La respuesta a esa pregunta se compara con el número de años estudiados por el trabajador.

iii) La medida empírica: se utiliza cuando no hay en la encuesta una pregunta sobre la educación requerida en el puesto de trabajo. En este caso se mide el desajuste educacional comparando el nivel de estudios del trabajador con la media, la moda y la desviación estándar de la educación requerida en su ocupación.

La aproximación a los desajustes educacionales no es la misma en función de la medida que se utilice, de hecho, los valores obtenidos mediante la medida

7 En la página web del Ministerio de Empleo y Seguridad Social se describe la metodología para la realización de la encuesta. Ver http://www.empleo.gob.es/estadisticas/ecvt/ Ecvt2010/ANE/Metodologia.htm 
empírica muestran valores más bajos en sobreeducación, que en el método objetivo y subjetivo (McGuinness, 2006). La literatura tampoco es concluyente respecto de la utilización de un método u otro, así autores como Verhaest y Omey (2006) prefieren el método objetivo y subjetivo que el empírico y, otros, como Hartog (2000), se decantan por el subjetivo. En términos prácticos, la elección de uno u otro método depende en la mayoría de los casos de la propia disponibilidad de datos.

En nuestro caso, la elección ha sido el método subjetivo debido a la propia formulación de la ECVT. De hecho, en la encuesta se les pregunta a los trabajadores:

- ¿Considera que el puesto de trabajo que ocupa es el correcto de acuerdo con la formación que usted tiene?

a) Es el correcto.

b) Es más bajo que mi formación.

c) Está por encima de mi formación.

d) Necesitaría una formación distinta a la que tengo.

Si la respuesta a esta cuestión es "el correcto" este trabajador se ha clasificado como adecuadamente educado (AE), si la respuesta es "más bajo" como infraeducado (IE), si la respuesta es "por encima" como sobreeducado (SE) y si la respuesta es "necesitaría una formación distinta", como un trabajador incorrectamente educado.

Respecto a la variable que hace referencia al desajuste en habilidades, utilizamos la siguiente pregunta de la ECVT:

- ¿En qué grado le sirve su formación académica para el trabajo que realiza?

La respuesta puede ser contestada en una escala del 0 a 10.

Si los trabajadores puntúan de 0 a 4 sus habilidades para el trabajo, consideramos que los trabajadores tienen competencias distintas a las exigidas por el puesto, o que tienen una inadecuada capacitación (IC), si la respuesta está entre 5 y 9 fueron clasificados como adecuada capacitación (AC) y si la respuesta es 10 se considera al trabajador sobrecapacitado $(\mathrm{SC})^{8}$. Se entiende que un trabajador que contesta 10 posee capacidades por encima de las necesarias porque normalmente un individuo que considera que sus habilidades se ajustan a su trabajo nunca contestará con la puntuación máxima. En la ECTV no se dispone de información más específica relativa a las habilidades del individuo que no haga referencia al proceso formativo. Como consecuencia, la interpretación de

8 Para mostrar la robustez de las estimaciones se ha modificado la elección de los umbrales, considerando que un trabajador está sobrecapacitado si su respuesta está entre 8 y 10. Los resultados de la variable sobrecapacitación son robustos a la nueva especificación, si bien la variable infracapacitación deja de ser significativa. Este resultado no es sorprendente, ya que la variable de referencia - estar adecuadamente cualificado- ha reducido su rango de valores y en media toma un valor inferior, por lo que las diferencias con los trabajadores infracapacitados no son tan significativas. 
TABLA 1

ESTADÍSTICAS DESCRIPTIVAS: AJUSTE EDUCACIONAL Y DE COMPETENCIAS

(Variables en porcentaje)

\begin{tabular}{|lllccc|}
\hline & & \multicolumn{4}{c|}{ Ajuste de competencias } \\
\cline { 3 - 6 } & & IC & AC & SC & Total \\
\hline \multirow{2}{*}{ Ajuste } & IE & 50,8 & 11,16 & 38,05 & 100 \\
educacional & AE & 58,04 & 18,01 & 23,95 & 100 \\
& SE & 44,71 & 9,10 & 46,19 & 100 \\
\hline
\end{tabular}

Nota: AE (adecuadamente educado), SE (sobreeducado), IE (infraeducado), AC (adecuadamente capacitado), SC (sobrecapacitado) e IC (infracapacitado).

Fuente: Elaboración propia a partir de ECVT.

esta variable no es comparable totalmente con los estudios previos ${ }^{9}$. En dichos estudios la variable que mide habilidades incluye tanto las proporcionadas durante el proceso formativo como las adquiridas a lo largo de la vida laboral (a través de su experiencia profesional en distintos trabajos o el propio aprendizaje por la práctica o learning by doing). Así, puede darse el caso de un individuo considere que su formación no le ha proporcionado las habilidades necesarias para su puesto de trabajo, pero las haya adquirido por otras vías. En el presente estudio este individuo sería calificado como infracapacitado, mientras que en otros podría tener otra consideración.

En la Tabla 1 se recogen los porcentajes de individuos que han declarado estar en una situación de desajuste/ajuste educacional y desajuste/ajuste en habilidades. La correspondencia entre educación y habilidades es moderada, lo que demuestra que ambas preguntas recogen realidades distintas. En torno al 50\% de los trabajadores consideran que padecen infraeducación e infracapacitación, cifra similar a la que mantienen los sobreeducados y sobrecapacitados. Si bien existen resultados paradójicos. El 58\% de los individuos con ajuste educacional tiene un problema de infracapacitación y 44,7\% de los individuos sobreeducados tiene habilidades inferiores a las exigidas por el puesto. De hecho, solo el 18 por cierto de los individuos que consideran que tienen un ajuste educacional afirman, asimismo, tener un ajuste de competencias.

La ECVT proporciona 19 facetas distintas de la satisfacción en el trabajo. En este trabajo nos vamos a centrar en la satisfacción laboral global, la satisfacción con el salario, con las horas trabajadas, con la promoción y con el tipo de trabajo. Las respuestas del trabajador pueden ser contestadas en una escala del 0 al 10. Los valores han sido agrupados en tres categoría: el valor 0 expresa baja

9 La pregunta sobre competencias que tiene la ECVT coincide prácticamente con la que se emplea en los trabajos que utilizan el Panel Europeo de hogares, si bien este último incorpora otra pregunta adicional preguntando al trabajador si con sus capacidades podría desarrollar un trabajo de mayor categoría. La definición de infracapacitado sería comparable con la de Badillo-Amador et al. (2012), ya que estos autores utilizan la misma pregunta para medir dicha variable, pero la de sobrecapacitado no lo sería. 
satisfacción (valores de 0 a 4), el 1 una satisfacción media (valores de 5 a 7 ) y el 2 alta (valores de 8 a 10) ${ }^{10}$. La finalidad de dicha agrupación es doble. De un lado, los resultados van a ser más sencillos de interpretar. De otro, se elimina parte del componente subjetivo que tiene una persona a la hora de asignar un valor concreto a su satisfacción laboral.

Además de las variables relacionadas con el ajuste educacional y de habilidades, se han tenido en cuenta otros factores condicionantes del bienestar del trabajador en su puesto de trabajo: aquellos propios del individuo (edad, sexo, antigüedad en el puesto de trabajo y ganancias de la unidad familiar a la que pertenece), las características objetivas del puesto (tipo de empleo y salario) y otras de carácter más subjetivo (grado de estabilidad, independencia en la toma de decisiones, estrés, desarrollo personal, toma de decisiones y percepción del entorno sociolaboral). La inclusión de estas últimas variables resulta de especial interés al medir la satisfacción laboral y supone una ventaja de la ECVT frente a otro tipo de encuestas que no disponen de esta información. Además permiten aislar los efectos diferenciales de los desajustes educacionales y de competencias.

\section{Modelo eCONOMÉtrico}

El modelo parte de una función de utilidad laboral individual para cada trabajador que adopta la expresión utilizada por Clark y Oswald (1996):

$$
u=u(x j, d l)
$$

donde $x$ incluiría aquellas variables relacionadas con las características individuales del trabajador, $j$ las referidas a las características del empleo, objetivas y subjetivas, y $d l$ las que consideran los desajustes educacionales y de habilidades.

Para la estimación del modelo se supone que la satisfacción laboral puede ser utilizada como proxy de la utilidad laboral individual, por lo que se propone el siguiente modelo:

$$
S L_{i}^{*}=\beta X_{i}+\alpha J_{i}+\gamma d l_{i}+\varepsilon_{i}
$$

La satisfacción laboral $\left(S L^{*}\right)$ es una variable latente que denota la probabilidad del individuo $i$ de estar satisfecho en el trabajo. Esta variable resulta inobservable, y para su medición se utiliza una valoración ordinal que realiza el propio individuo. La relación entre la variable latente y nuestra variable de satisfacción laboral se muestra por la siguiente expresión:

10 El criterio utilizado es estándar y corresponde al seguido por el trabajo de Gamero (2004), quien utiliza, asimismo, la ECVT. De esta forma, los resultados entre ambos trabajos podrían ser comparables para el lector. Se han realizado, asimismo, análisis de robustez incluyendo exclusivamente como individuos satisfechos aquellos que respondían esta respuesta con valores entre 9-10. Los resultados son robustos en el caso de sobre e infraeducación y la sobrecapacitación. La variable infracapacitación pierde significatividad. Este resultado no es de extrañar, porque esta variable es muy sensible a cualquier modificación en su medición, tal y como muestra el trabajo de Allen y Van der Velden (2011). 


$$
S L_{i}=\left[\begin{array}{cccc} 
& & & S L_{i}^{*} \leq \mu_{0} \\
0 & \text { si } & \\
1 & \text { si } & \mu_{0}<S L_{i}^{*} \leq \mu_{1} \\
2 & \text { si } & \mu_{1}<S L_{i}^{*} \leq \mu_{2} \\
& & & \\
& & & \\
& & \cdots & \\
10 & \text { si } & \mu_{10} \leq S L_{i}^{*}
\end{array}\right]
$$

donde $\mu$ son los valores de la satisfacción laboral latente que definen los intervalos de la satisfacción laboral observada. Se supone que $\mu_{0}=0$.

Debido a que los valores de la variable dependiente están ordenados, en la estimación del modelo se ha utilizado un modelo probit ordenado. Para la interpretación de los resultados se estiman los cambios marginales de cada categoría, de forma que se refleje la modificación en las probabilidades a través de la estimación de los cambios marginales de cada variable explicativa. El efecto marginal corresponde a la pendiente de la curva que relaciona la variable explicada $x_{i}$ con la probabilidad de que la satisfacción laboral observada tome el valor $j$ condicionada a $x_{i}$, manteniendo el resto de las variables constantes. En definitiva, la curva que relaciona $x_{i}$ con $\operatorname{Pr}\left(S L_{i}=j \mid x_{i}\right)$, siendo $j=0,1,2$, dado que nuestra variable sólo toma tres valores. Los resultados que se muestran son los efectos marginales cuando la satisfacción laboral toma el valor máximo (2) ${ }^{11}$.

\section{Resultados}

Las Tablas 2 y 3 muestran una descripción de los desajustes educacionales y de competencias en función del sexo, la edad y el nivel educacional de los trabajadores. El porcentaje de hombres que presentan un correcto ajuste educacional es superior al de las mujeres ( $82 \%$ frente a $76 \%)$. La situación es la contraria si se considera el porcentaje de trabajadores con sobreeducación, es decir, son las mujeres las que mayor porcentaje presentan comparadas con los varones. Este resultado pudiera explicarse, en parte, por la elevada demanda de estudios universitarios realizada en España en los últimos años por parte de las mujeres, considerablemente superior a la de los varones. Por otra parte, la movilidad de las mujeres podría ser más baja que la de los varones, ya que en muchos casos asumen obligaciones familiares o relacionadas con el hogar y supeditarían su participación en el mercado de trabajo a la de su cónyuge. No ha de obviarse, por último, la posibilidad de que la promoción de las mujeres sea más dificultosa que la de los varones como consecuencia de una posible discriminación por razón de sexo. Como consecuencia, la probabilidad de que una mujer ocupe un puesto con un nivel educacional inferior al exigido puede ser superior a la del varón.

11 Dirigirse a los autores para solicitar las estimaciones correspondientes a los valores 0 y 1 . 
TABLA 2

ESTADÍSTICAS DESCRIPTIVAS: AJUSTE EDUCACIONAL

(Variables en porcentaje)

\begin{tabular}{|lrrrr|}
\hline & AE & SE & IE & Total \\
\hline Sexo & & & & \\
Masculino & & & & \\
Femenino & 82,72 & 14,83 & 2,45 & 13.564 \\
Edad & 76,42 & 21,78 & 1,80 & 9.644 \\
$16-30$ & & & & \\
$31-40$ & & & & \\
$41-50$ & 73,44 & 24,02 & 2,54 & 3.976 \\
$51-60$ & 77,19 & 20,78 & 2,02 & 6.625 \\
$>60$ & 81,30 & 16,45 & 2,25 & 6.989 \\
& 86,13 & 11,89 & 1,99 & 4.584 \\
Educación & 89,56 & 8,22 & 2,22 & 1.034 \\
(1) Sin estudios o inferior a E. primarios & & & & \\
(2) E. primarios & & & & \\
(3) E. secundarios & 93,67 & 3,59 & 2,74 & 948 \\
(4) Formación profesional & 90,10 & 7,30 & 2,60 & 4.304 \\
(5) E. universitarios (3-5 años) & 85,40 & 12,45 & 2,15 & 4.746 \\
\hline Total & 75,73 & 21,52 & 2,75 & 7.454 \\
& 71,68 & 27,26 & 1,06 & 5.756 \\
\hline
\end{tabular}

Nota: AE (adecuadamente educado), SE (sobreeducado) e IE (infraeducado).

Fuente: Elaboración propia a partir de ECVT.

Por lo que respecta a la edad, los individuos más jóvenes son los que presentan un mayor desajuste educacional, al estar desempeñando puestos que requieren un nivel educacional inferior al que poseen. Hay que tener en cuenta que el proceso de ajuste entre la formación alcanzada y el puesto de trabajo se desarrolla a lo largo del tiempo y que, por tanto, los individuos más jóvenes tienen mayores probabilidades de estar realizando un puesto de trabajo que no corresponda a su nivel formativo. Hay que tener en consideración que las exigencias en el puesto de trabajo no son exclusivamente educativas sino de competencias, y estas últimas se adquieren, en parte, por la experiencia.

Por último, en relación con el nivel educacional, tal y como cabría esperar, a medida que se incrementan los años de estudio, mayor es la probabilidad de estar en un empleo con nivel exigido inferior al que posee el trabajador.

En la Tabla 3 se recogen, asimismo, una descripción de los resultados atendiendo a los niveles de habilidades adquiridos en el proceso de formación. Los varones presentan un mayor ajuste de competencias que las mujeres. Por lo que respecta a la edad, llama la atención que mientras que los individuos más jóvenes tienen en promedio un nivel educacional superior al correspondiente a 
su puesto de trabajo, desde el punto de vista de las competencias los resultados son inversos. De hecho, el porcentaje de individuos con habilidades inferiores a las exigidas en su puesto de trabajo decrece con la edad de forma lineal. Es razonable pensar que a medida que se incrementa la edad del trabajador se incremente su experiencia y las competencias necesarias para realizar su trabajo eficientemente, de forma que nuestra variable de competencias no solamente estaría recogiendo las competencias adquiridas con la formación, sino también las adquiridas con la experiencia. Este resultado avala, de nuevo, la diferencia existente entre cada una de las preguntas planteadas.

\section{TABLA 3}

ESTADÍSTICAS DESCRIPTIVAS: AJUSTE DE COMPETENCIAS

(Variables en porcentaje)

\begin{tabular}{|c|c|c|c|c|}
\hline & $\mathrm{AC}$ & $\mathrm{SC}$ & IC & Total \\
\hline \multicolumn{5}{|l|}{ Sexo } \\
\hline Masculino & 56,80 & 14,52 & 28,68 & 13.548 \\
\hline Femenino & 53,71 & 18,77 & 27,52 & 9.633 \\
\hline \multicolumn{5}{|l|}{ Edad } \\
\hline $16-30$ & 52,41 & 14,79 & 32,80 & 3.976 \\
\hline $31-40$ & 55,74 & 15,81 & 28,45 & 6.622 \\
\hline $41-50$ & 56,19 & 16,98 & 26,83 & 6.984 \\
\hline $51-60$ & 57,31 & 16,66 & 26,02 & 4.573 \\
\hline$>60$ & 53,51 & 18,71 & 27,78 & 1.026 \\
\hline \multicolumn{5}{|l|}{ Educación } \\
\hline (1) Sin estudios o inferior a E. primarios & 42,02 & 8,68 & 49,30 & 933 \\
\hline (2) E. primarios & 53,35 & 9,74 & 36,92 & 4.304 \\
\hline (3) E. secundarios & 54,61 & 9,71 & 35,67 & 4.746 \\
\hline (4) Formación profesional & 58,65 & 14,38 & 26,97 & 7.442 \\
\hline (5) E. universitarios (3-5 años) & 56,01 & 30,30 & 13,69 & 5.756 \\
\hline Total & 55,52 & 16,28 & 28,2 & 23.181 \\
\hline
\end{tabular}

Nota: AC (adecuadamente capacitado), SC (sobrecapacitado) e IC (infracapacitado). Fuente: Elaboración propia a partir de ECVT.

A medida que se incrementan los años de estudio el porcentaje de trabajadores con ajuste de habilidades se incrementa. Los estudios superiores colaboran, por tanto, positivamente en la adquisición de competencias dirigidas a ejercer un puesto de trabajo. De hecho, son los individuos con estudios universitarios los que menor desajuste de competencias parecen encontrar (13,6\%), mientras que el porcentaje es casi el doble para aquellos que han cursado formación profesional o bachillerato. 
A continuación se muestran los resultados econométricos. En primer lugar nos detenemos en estudiar la influencia de estas variables en la satisfacción laboral (Tabla 5). Posteriormente, analizaremos la influencia de estos desajustes sobre la satisfacción con el salario y el número de horas trabajadas (Tabla 6) y, por último, la satisfacción con la promoción y el tipo de trabajo (Tabla 7) ${ }^{12}$. Cabría preguntarse si estas variables están midiendo matices distintos de la satisfacción o por lo contrario recogen una misma realidad, en cuyo caso no tendría sentido realizar las estimaciones por separado. Por esta razón se ha realizado un análisis de correlación entre las variables dependientes (Tabla 4). Como se puede observar, y tal y como cabría esperar, el grado de correlación es positivo, si bien no muy elevado, lo que evidencia que cada variable de satisfacción valora matices distintos del puesto de trabajo. Por este motivo resulta de interés un análisis desagregado de cada satisfacción.

TABLA 4

COEFICIENTE DE CORRELACIÓN ENTRE LAS VARIABLES

DE SATISFACCIÓN

\begin{tabular}{|lccccc|}
\hline \multicolumn{1}{|c}{ Satisfacción } & $\begin{array}{c}\text { En el } \\
\text { trabajo }\end{array}$ & $\begin{array}{c}\mathrm{N}^{\mathrm{o}} \\
\text { de horas }\end{array}$ & Salario & Promoción & $\begin{array}{c}\text { Tipo de } \\
\text { actividad }\end{array}$ \\
\hline En el trabajo & 1,0000 & & & & \\
$\mathrm{~N}^{\text {o de horas }}$ & 0,4367 & 1,0000 & & & \\
Salario & 0,4570 & 0,3249 & 1,0000 & & \\
Promoción & 0,2966 & 0,1650 & 0,2478 & 1,0000 & \\
Tipo de actividad & 0,5505 & 0,3060 & 0,2913 & 0,1958 & 1,0000 \\
\hline
\end{tabular}

Fuente: Elaboración propia a partir de ECVT.

Como variables independientes, además de las variables relativas al ajuste educacional y de competencias, se recogen las características personales y las características objetivas y subjetivas del trabajo, así como una serie de variables de control. Entre las primeras se incluye la edad, la antigüedad (medida como los años que llevan en la empresa), el sexo, el estado civil y las ganancias del hogar (la medición de esta variable se hace por tramos de acuerdo con la encuesta). En las características objetivas del puesto se considera si el trabajador es autónomo, si ocupa un nivel directivo, intermedio o bajo, el salario (medido por tramos), las horas trabajadas, el tipo de jornada (parcial o total) y si trabaja en empresa

12 Como se ha señalado, todas las variables dependientes: satisfacción con el trabajo, con el salario, con el número de horas trabajadas, con la promoción y con el tipo de trabajo se han obtenido por medio de una pregunta en la encuesta que toma valores entre 0 y 10 . Estos valores se han agrupado en tres categorías: bajo (si los valores son por debajo del 5 ), medio (igual o mayor que 5 y menor que 8 ) y alto (igual o mayor que 8 ). 
privada o pública. Por último, como características subjetivas se incluyen el nivel de estrés del trabajo que desempeña, la estabilidad, el nivel de responsabilidad en la toma de decisiones y el ambiente laboral (estas características subjetivas se miden con una dummy). Dentro de las variables de control se incluyen variables dummy anuales y regionales (por comunidades autónomas).

Se distinguen así tres modelos. El modelo (1) incluye exclusivamente las características objetivas, el ajuste educacional y el de competencias, el modelo (2) incluye las características subjetivas del trabajo y de ajuste educacional y el (3) todas las variables. El objetivo fundamental de utilizar los tres modelos es comprobar la robustez de las variables relacionadas con el ajuste educacional y de habilidades, ya que pudieran estar correlacionadas con alguna variable explicativa.

En la Tabla 5 se muestran los resultados correspondientes a la satisfacción en el trabajo. Como se puede observar, la edad influye negativamente sobre la satisfacción en el trabajo. Se ha estudiado si pudiera existir una relación de U invertida, de forma que la satisfacción crezca hasta determinada edad y, posteriormente, decrezca, si bien los resultados no parecen apoyar esta hipótesis, debido a la falta de significancia de la variable edad al cuadrado. Por lo que respecta a la antigüedad, los resultados muestran que a medida que se incrementa esta variable también lo hace la satisfacción en el trabajo. Los resultados correspondientes al sexo no avalan, sin embargo, la existencia de diferencias significativas en la satisfacción de las mujeres respecto de los hombres. Por último, como cabría esperar, las ganancias en el hogar actúan de forma positiva sobre la satisfacción. Es factible que muchos de los problemas del trabajo pueden relativizarse si se tiene una fuente de ingresos alternativa.

En cuanto a las características objetivas del trabajo, el ejercer un puesto de trabajo de alto rango influye positivamente en el grado de satisfacción. Concretamente, los individuos que ocupan cargos de dirección se muestran entre $15,3 \%$ y $16,4 \%$ más satisfechos que los que no tienen cargos de responsabilidad. En el caso de los mandos intermedios también se observa esta ganancia en satisfacción, pero de menor cuantía (entre el 5,5\% y el 7,5\%). Los trabajadores autónomos también muestran ganancias en satisfacción, pero dichas ganancias se reducen considerablemente cuando se introducen las características subjetivas del trabajo, lo que implica una alta correlación entre ambos tipos de variables (especialmente, estabilidad e independencia). Por último, y en línea con la literatura existente, los salarios individuales actúan de forma positiva sobre la satisfacción, igual que los salarios del hogar. No obstante, los salarios del trabajador tienen mayor peso en la satisfacción laboral. Los trabajadores con salarios más altos tienen una probabilidad mayor de estar más satisfechos con su trabajo (entre $2,8 \%$ y $3 \%$ ).

La importancia que tienen las características subjetivas del trabajo en el análisis de la satisfacción laboral se hace patente en nuestro trabajo al exhibir algunas de estas variables los coeficientes estimados más altos. El hecho de tener un trabajo estable eleva en más de $22 \%$ la probabilidad de estar satisfechos con nuestro trabajo, algo similar ocurre si tenemos un ambiente laboral agradable (24\%). Asimismo, si el trabajo que se ejerce nos permite desarrollarnos personalmente supondrá un incremento de la probabilidad de satisfacción laboral en más de $30 \%$. Por el contrario, el estrés baja la probabilidad de satisfacción entre $10,5 \%$, aproximadamente. 


\section{TABLA 5}

SATISFACCIÓN EN EL TRABAJO

Modelo Probit ordenado. Efectos marginales para el valor máximo $(=2)$

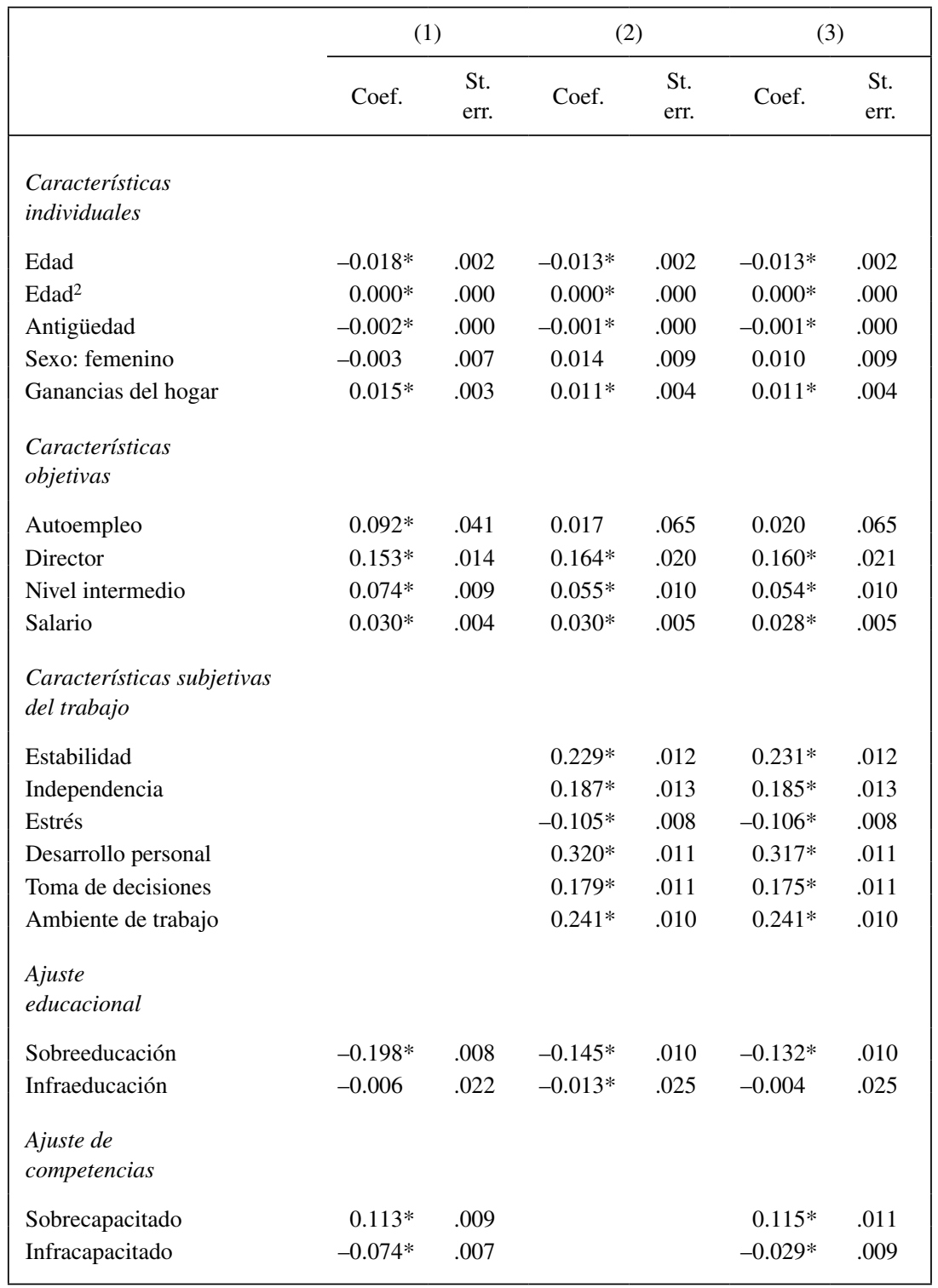

(*) Significativo al 5\%.

Fuente: Elaboración propia a partir de ECVT. 
A continuación se procede a analizar los desajustes educacionales y compentenciales, principal objetivo del artículo. Las estimaciones que recogen ambos desajustes avalan alguna de las hipótesis planteadas. El hecho de tener un nivel educacional superior al exigido en el puesto afecta de forma negativa a la satisfacción en el trabajo, bajando entre 13,2\% y 19,8\% la probabilidad de estar satisfecho. Las propias expectativas que genera un individuo cuando invierte tiempo y dinero en formarse hacen que la educación afecte negativamente a la satisfacción laboral. Tener una educación inferior a la exigida por el puesto también reduce la probabilidad de estar satisfecho, pero su impacto es menor (entre $0,4 \%$ y $1,3 \%$ ). Nótese, en todo caso, que esta última variable pierde significatividad al incluir en la estimación las variables relacionadas con el ajuste de competencias. Se observa, asimismo, la importancia de incluir las variables subjetivas, porque su omisión sobrevalora los coeficientes de los desajustes.

Por lo que respecta a los desajustes de competencias, los individuos sobrecapacitados tienen una probabilidad en torno al $11 \%$ superior de estar satisfechos en su puesto. Este resultado contrasta con el obtenido por el desajuste educacional, ya que los individuos sobreeducados se mostraban más insatisfechos que el resto. La explicación de esta aparente discrepancia se puede encontrar en las expectativas generadas por el trabajador. Un trabajador con sobreeducación estará más insatisfecho, pues es factible que esperase poder encontrar un trabajo de nivel educacional superior, mientras que uno sobrecapacitado no se ha generado una expectativa tan alta. De hecho, puede estar satisfecho al desempeñar adecuadamente su trabajo. Ahora bien, si el trabajador no tiene las capacidades necesarias para el correcto desempeño de su trabajo puede sentirse estresado por la dificultad que entraña su desarrollo y, por tanto, más insatisfecho.

En la Tabla 6 aparecen los resultados econométricos utilizando como variables dependientes la satisfacción con el salario ${ }^{13}$ y el número de horas trabajadas. Como se puede observar, los resultados correspondientes a la primera variable presentan fuertes similitudes a la satisfacción general en el trabajo. Igual que ocurría con la satisfacción en el trabajo, la sobreeducación influye negativamente sobre la satisfacción en el pago, disminuyendo la probabilidad de estar satisfecho con el salario entre $9,8 \%$ y $11,9 \%$ (un impacto algo más bajo que el que se obtiene en el caso de la satisfacción laboral en términos globales). En cuanto a los individuos infraeducados se observa un cambio importante respecto de los resultados de la Tabla 5, ya que mientras que su probabilidad de estar satisfechos globalmente es baja, su satisfacción respecto del salario es elevada. Es de esperar que dicho trabajador esté cobrando un salario por encima del que podría haber alcanzado en caso de que le hubiesen ubicado en un puesto acorde a su nivel educacional.

Respecto del ajuste de habilidades, los resultados son análogos a los relativos a la satisfacción general. La probabilidad de que un individuo esté satisfecho con su salario aumenta entre $6,4 \%$ y $7,3 \%$ si está sobrecapacitado. Por el contrario, dicha probabilidad se reduce entre $1,2 \%$ y $4,8 \%$ si el trabajador está infracapacitado.

13 Nótese que en las estimaciones correspondientes a la satisfacción con el salario se han excluido las variables explicativas relacionadas con el salario y las ganancias del hogar, para evitar problemas de colinealidad. 


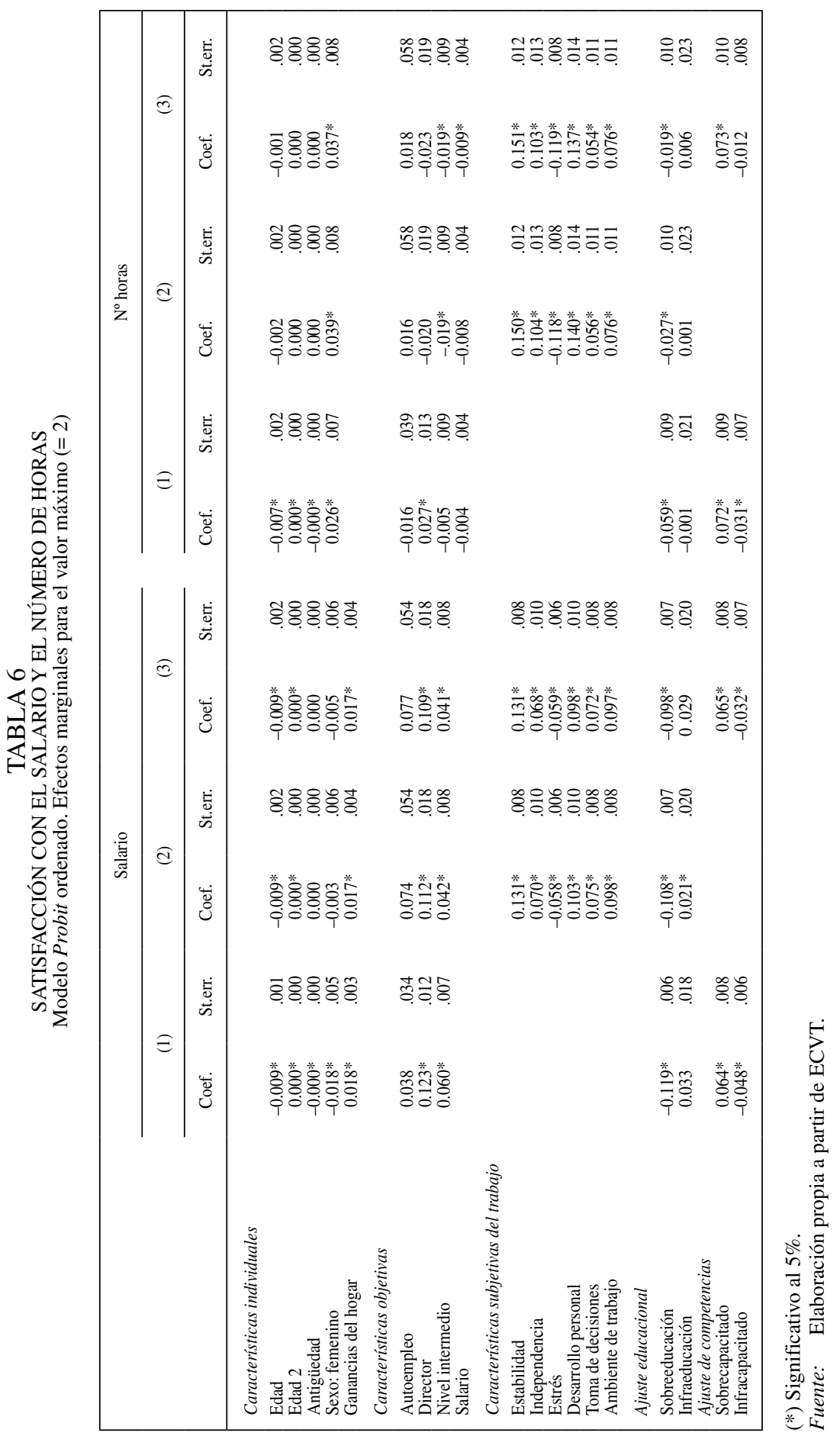


Por lo que respecta a la satisfacción con el número de horas trabajadas, de nuevo los individuos sobreeducados tienen mayor probabilidad de estar insatisfechos con el número de horas trabajadas, si bien la magnitud del impacto de esta variable es más pequeño y presenta un mayor margen de oscilaciones en los tres modelos considerados (la probabilidad de estar satisfecho con el horario laboral se reduce entre $5,9 \%$ y $1,9 \%$ si consideramos el modelo (1) y (3), respectivamente). Los resultados respecto de los trabajadores con nivel educacional inferior al exigido no son significativos.

En cuanto al ajuste de habilidades, los trabajadores sobrecapacitados tienen mayor probabilidad de estar satisfechos con su jornada laboral (en torno al 7,3\%). El hecho de tener habilidades inferiores influye negativamente en la satisfacción con el número de horas, probablemente porque, o bien les resulte insuficiente su jornada para realizar el trabajo eficientemente, o bien tenga que dedicar un número de horas relativamente más alto que sus compañeros para conseguir los mismos resultados. De hecho, cuando consideramos el modelo (3) en el que se incluyen variables más subjetivas como el estrés, se observa que el coeficiente de esta variable se reduce considerablemente, lo que evidencia la relación existente entre la falta de habilidades con la necesidad de más horas de trabajo y con el consiguiente estrés laboral. No ocurre lo mismo con la sobrecapacitación que mantiene el valor de su coeficiente y no se ve afectado por la introducción de las variables subjetivas.

Por último, en la Tabla 7 se toman como variables dependientes la satisfacción con las posibilidades de promoción dentro de la empresa y con el tipo de trabajo realizado. El trabajador con sobreeducación es más probable que esté menos satisfecho con la promoción dentro de su empresa (entre 4,8\% y 7,9\%). Es coherente que un individuo cuyo nivel educacional sea superior al exigido en el puesto considere que su talento está desaprovechado y esté menos conforme con el mecanismo de promoción de su empresa. La influencia de la variable infraeducación es positiva, si bien no es significativa.

Los resultados respecto del ajuste de habilidades muestran que la sobrecapacitación no afecta al grado de satisfacción con la promoción. El coeficiente estimado no es significativo y su magnitud muy reducida. Sin embargo, los trabajadores infracapacitados ven reducida 5,7\% su probabilidad de estar satisfechos con la promoción si lo comparamos con los que no presentan desajuste de competencias (incluyendo las características objetivas).

La satisfacción con el tipo de trabajo a desempeñar emerge como uno de los puntos importantes en el estudio de la satisfacción laboral y los desajustes educacionales y de competencias a juzgar por la importancia de los coeficientes estimados obtenidos en ambos desajustes. En el caso del primero, los trabajadores sobreeducados presentan una probabilidad de estar satisfechos más baja que los trabajadores sin desajuste (entre $14 \%$ y $21 \%$ ). Nótese que las cifras son más elevadas que las correspondientes a las anteriores tablas, lo que evidencia que al trabajador no solo le importa el salario, la jornada o la promoción, sino otras cuestiones más sutiles, como el carácter más o menos repetitivo, creativo o interesante del puesto. Los trabajadores infraeducados obtienen una probabilidad más alta de estar satisfechos con el tipo de trabajo, si bien el valor es muy pequeño (entre $0,4 \%$ y $1,3 \%$ ) y no significativo.

En cuanto al desajuste de competencias, el efecto resulta ser muy similar al obtenido para el caso de la satisfacción laboral global, si bien destaca el alto 


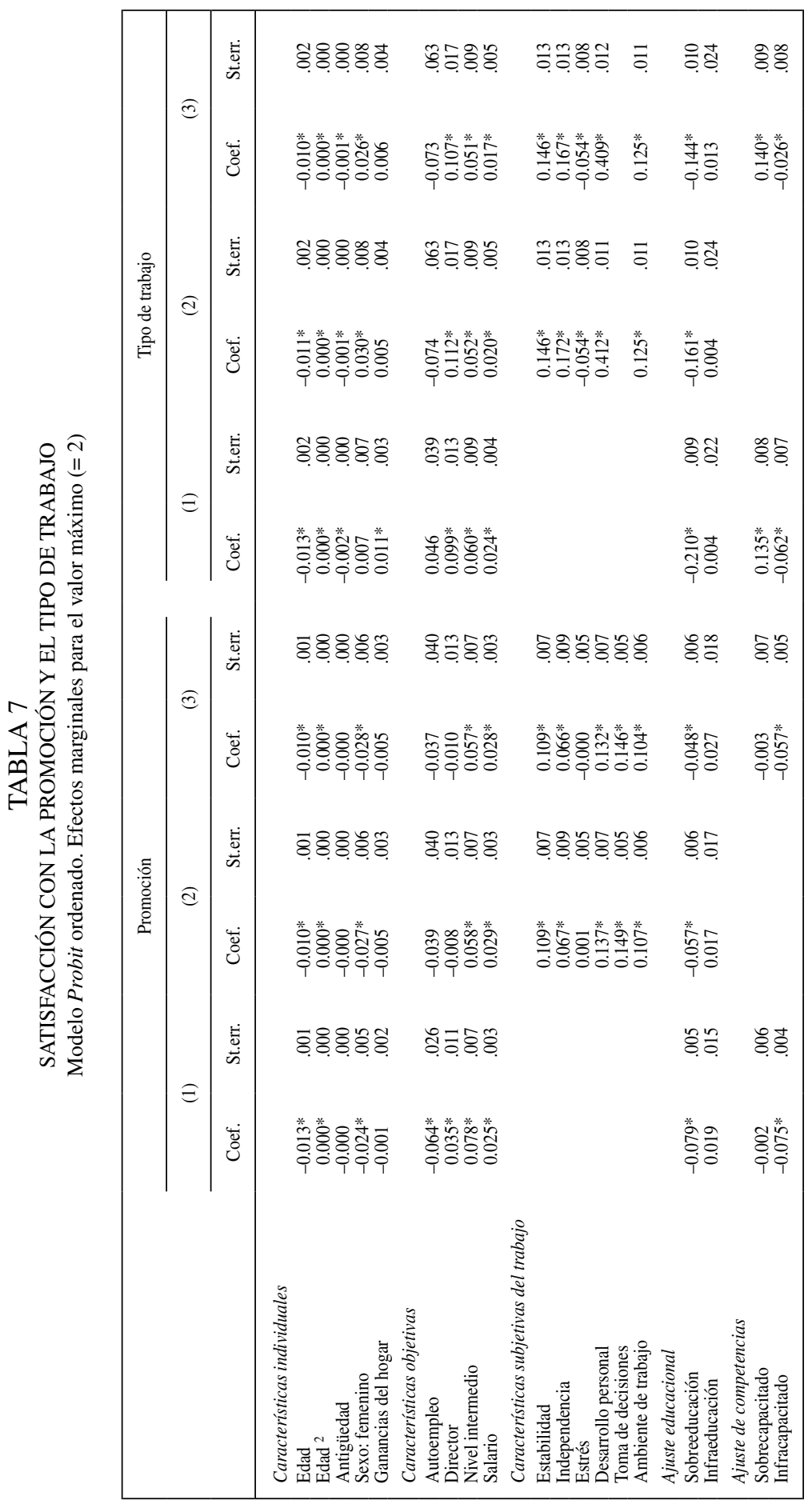

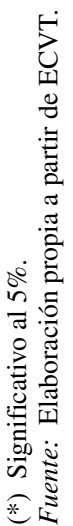


coeficiente obtenido en el caso de los trabajadores sobreeducados que presentan una mayor probabilidad de estar satisfechos con el tipo de tareas desempeñadas (por encima del 13\%). Lo contrario se observa en el caso de los trabajadores infraeducados, en los que su probabilidad de satisfacción se reduce oscilando entre $6,2 \%$ y $2,6 \%$ en el modelo (1) y (3), respectivamente.

\section{Conclusiones}

El artículo utiliza un modelo teórico que mide la repercusión de los desajustes educacionales y de habilidades obtenidas por el proceso formativo en la satisfacción laboral y procede a estimarlo a través de un modelo probit ordenado. Además de las variables relacionadas con dichos desajustes se incluyen otras relacionadas con las propias características del individuo (edad, sexo, antigüedad en el puesto de trabajo y ganancias de la unidad familiar a la que pertenece) y del trabajo, ya sean estas objetivas (tipo de empleo y salario) o de carácter más subjetivo (grado de estabilidad, independencia, estrés, desarrollo personal, toma de decisiones en el trabajo y percepción del entorno laboral). Las variables educativas y de competencias se miden mediante el procedimiento subjetivo, basándose en las respuestas que ofrece el trabajador.

Los resultados muestran cómo la sobreeducación es una fuente clara de insatisfacción en el puesto de trabajo. Los individuos con un nivel educacional superior al exigido por su puesto tienen menor probabilidad de estar satisfechos en su trabajo, a nivel general, respecto del pago que perciben, al número de horas que trabajan, a las posibilidades de promoción dentro de su empresa u organización y respecto del tipo de puesto que realizan. Si la satisfacción constituye un elemento clave para la productividad del trabajador, tener a un individuo sobreeducado implicará, además de una asignación ineficiente de recursos, una reducción del potencial productivo de la empresa.

Los individuos con un nivel educacional inferior al exigido por el puesto suponen, asimismo, una ineficiente asignación de recursos, si bien su satisfacción no es significativamente inferior a la de aquellos cuyo ajuste educacional es adecuado, independientemente de si se considera la satisfacción global, lo relacionado con el pago, con el número de horas trabajadas, las posibilidades de promoción y el tipo de actividades desarrolladas.

Los resultados correspondientes al ajuste de habilidades difieren respecto de los educacionales, tal y como se intuía en el propio análisis descriptivo. Si el proceso formativo nos ha proporcionado habilidades o competencias elevadas la probabilidad de satisfacción laboral va a ser superior tanto en términos globales como con el número de horas trabajadas y con el tipo de trabajo desarrollado. Un trabajador con pocas habilidades presenta el efecto contrario. Esta idea contrasta con los resultados obtenidos en otros trabajos en los que se muestra una relación negativa entre sobrecapacitación y satisfacción laboral. Esta discrepancia es, en parte, atribuible a la diferente medición de la variable, ya que en nuestro estudio la sobrecapacitación que se tiene en cuenta es la que fue provocada por la formación recibida por el individuo, mientras que en otros trabajos puede ser provocada por una mayor experiencia o por el conocimiento por la práctica adquirida por el trabajador. La encuesta utilizada en este trabajo no dispone de una medida más amplia de las capacidades o habilidades del trabajador. 
Además, y como se ha señalado, ambas medidas de desajuste han sido captadas mediante el método subjetivo, por lo que solo serían estrictamente comparables con aquellos estudios que hayan utilizado el mismo método.

En cualquier caso, y debido a los resultados obtenidos, resultaría sumamente conveniente que las empresas a la hora de diseñar sus organigramas, y sobre todo realizar los procesos de selección de personal, tengan en cuenta no solo el nivel educacional, sino el de competencias. Un mal ajuste laboral tiene un efecto sustancial en la satisfacción laboral y, por ende, lo tendrá en la productividad de la empresa.

\section{REFERENCIAS}

Albert, Cecilia y Mallorquí, Nuria (2011). "Desajuste educacional en el mercado de trabajo, un futuro próximo", en Investigaciones de Economía de la Educación 6, AEAD 666-676.

Allen, Jim y Van der Velden, Rolf (2001). "Educational Mismatches versus Skill Mismatches: Effects on Wages, Job Satisfaction, and On-the-Job Search", Oxford Economic Paper, vol. 53 (3): 434-452.

Badillo, Amador; Lourdes, Ángel; López, Nicolás y Vila L. E., Luis E. (2012). "The consequences on job satisfaction of job-worker educational and skill mismatches in the Spanish labour market: a panel analysis", Applied Economics Letters (1350-4851), March, 19 (4), 319.

Battu, Harminder; Belfield, Clive R. y Sloane, Peter J. (1999). "Overeducation Among Graduates: A Cohort View”, Education Economics, 17 (1); 21-38.

Budría, Santi. y Moro-Egido, Ana I. (2009). "The Overeducation Phenomenos in Europe", Revista International de Sociología, 67 (2): 329-345.

Cabral, José A. (2005). "Skill mismatches and job satisfaction", Economic Letters, 89, pp. 39-47.

Clark, Andrew E. y Oswal, Andrew J. (1996). "Satisfaction and comparison income", Journal of Public Economics, 61: 359-381.

Fabra, Eugenia y Camisón, Cecilia (2008). “Ajuste entre el capital humano del trabajador y su puesto de trabajo como determinante de la satisfacción laboral", Revista del Ministerio de Trabajo e Inmigración, 76, octubre; 129-142.

Freeman, Richard (1976). The overeducated American, New York, Academic Press. Fleming, Christopher M. y Kler, Parvinder (2007). "I'm Too Clever for this Job: A Bivariate Probit Analysis on Overeducation and Job Satisfaction in Australia", Applied Economics, 40 (9): 1123-1138.

Gamero Burón, Carlos (2004). "Satisfacción laboral de los asalariados en España. Especial referencia a las diferencias de género", Cuadernos de Economía, vol. 27, 109-146.

García Montalvo, José (1995). "Occupation and overqualification: the Spanish case”, FEDEA Working Paper $\mathrm{N}^{\circ}$ 95-20: Madrid.

García Serrano, Carlos y Malo, Miguel A. (1996). "Desajuste educacional y movilidad laboral en España”, Revista de Economía Aplicada, IV(11); 105-131.

Green, Francis. y McIntosh, Steven (2007). "Is There a Genuine Under-utilization of Skills Amongst the Over-qualified?” Applied Economics, 39: 427-439. 
Green, Francis. y Zhu, Yu (2010). "Overqualification, Job Dissatisfaction, and Increasing Dispersion in the Returns to Graduate Education", Oxford Economic Papers 62 (4): 740-763.

Hartog, Joop (2000). "Over-education and earnings: where are we, where should we go?", Economics of Education Review, 19: 131-147.

Hersch, Joni, (1991). "Education Match and Job Match", The Review of Economics and Statistics, MIT Press, 73(1); 140-44.

Mavromaras, Kostas; McGuinness, Seamus y Fok, Yin King (200). “Assessing the Incidence and Wage Effects of Overskilling in the Australian Labour Market", The Economic Record, 85 (268): 60-72.

Mavromaras, Kostas, McGuinness, Seamus y Wooden, Mark (2007). "Overskilling in the Australian Labour Market", Australian Economic Review, 40 (3): 307-312.

McGuinness, Seamus (2003). "Overeducation as a Sheepskin effect: Evidence from Northern Ireland", Applied Economics, 35 (5): 597-608.

McGuinness, Seamus (2006). "Overeducation in the Labour Market", Journal of Economic Survey, 20 (3): 387-417.

McGuinness, Seamus y Sloane, Peter J. (2011). "Labour Market Mismatch Among UK Graduates: An Analysis Using REFLEX Data", Economics of Education Review, 30 (1): 130-145.

Peiró, José M. Agut, Sonia y Grau, Rosa (2010). "The Relationship Between Overeducation and Job Satisfaction Among Young Spanish Workers: The Role of Salary, Contract of Employment, and Work Experience", Journal of Applied Social Psychology, 40, 3: 666-689.

Pérez P., F.; Serrano, L.; Uriel, E. y Mas, M. (1998). Capital humano, crecimiento económico y desarrollo regional en España (1964-1997), Fundación Bancaja.

Tsang, Mun C. (1987). "The impact of under-utilization of education on productivity", Economics of Education Review, 6 (3): 239-254.

Tsang, Mun C. y Levin, Henry M. (1985). "The economics of overeducation", Economics of Education Review, 4 (82): 93-104.

Verhaest, Dieter y Omey, Eddy (2006). "The Impact of Overeducation and its Measurement," Social Indicators Research, Springer, vol. 77 (3): 419-448. 
